\title{
The orbits of the Hurwitz action of the braid groups on the standard generators
}

by

\author{
Yoshiro Yaguchi (Hiroshima)
}

\begin{abstract}
The Hurwitz action of the $n$-braid group $B_{n}$ on the $n$-fold direct product $\left(B_{m}\right)^{n}$ of the $m$-braid group $B_{m}$ is studied. We show that the orbit of any $n$ - tuple of the $n$ standard generators of $B_{n+1}$ consists of the $(n-1)$ th powers of $n+1$ elements.
\end{abstract}

1. Introduction. The $n$-braid group, denoted by $B_{n}$, has the following presentation [1, 3]:

$$
\left\langle\sigma_{1}, \ldots, \sigma_{n-1} \mid \begin{array}{ll}
\sigma_{i} \sigma_{j} \sigma_{i}=\sigma_{j} \sigma_{i} \sigma_{j} & (|i-j|=1) \\
\sigma_{i} \sigma_{j}=\sigma_{j} \sigma_{i} & (|i-j|>1)
\end{array}\right\rangle,
$$

where $\sigma_{i}$ is the $i$ th standard generator represented by a geometric $n$-braid depicted in Figure 1.1.

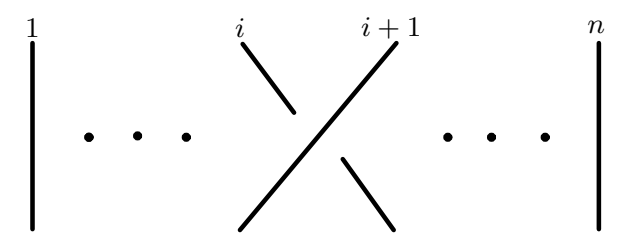

Fig. 1.1

Let $G$ be a group. The following action of $B_{n}$ on the $n$-fold product $G^{n}$ of $G$ is called the Hurwitz action.

Definition 1.1. The Hurwitz action of $B_{n}$ on $G^{n}$ is the right action defined by

$$
\begin{aligned}
\left(g_{1}, \ldots, g_{i-1}, g_{i}, g_{i+1},\right. & \left.g_{i+2}, \ldots, g_{n}\right) \cdot \sigma_{i} \\
& =\left(g_{1}, \ldots, g_{i-1}, g_{i+1}, g_{i+1}{ }^{-1} g_{i} g_{i+1}, g_{i+2}, \ldots, g_{n}\right),
\end{aligned}
$$

where $\sigma_{1}, \ldots, \sigma_{n-1}$ are the standard generators of $B_{n}$.

2010 Mathematics Subject Classification: Primary 20F36; Secondary 20F05.

Key words and phrases: braid group, Hurwitz action, standard generators. 
In this paper, we denote the orbit of $\left(g_{1}, \ldots, g_{n}\right) \in G^{n}$ under the Hurwitz action of $B_{n}$ by $\left(g_{1}, \ldots, g_{n}\right) \cdot B_{n}$.

There is a strong relationship between the Hurwitz actions of $B_{n}$ on $G^{n}$ and the equivalence classes of braided surfaces when $G$ is a braid group [7, 8, 9, 5, 10].

We study the Hurwitz action of $B_{n}$ on $\left(B_{n+1}\right)^{n}$, so $G=B_{n+1}$. Throughout this paper, we use the symbol " $s_{i}$ " to denote the $i$ th standard generator of $B_{n+1}$, and " $\sigma_{i}$ " to denote that of $B_{n}$.

In [4, S. P. Humphries proved the following.

Theorem $1.2([4])$. The orbit $\left(s_{1}, \ldots, s_{n}\right) \cdot B_{n}$ consists of $(n+1)^{n-1}$ elements.

The following is our main result.

Main Theorem 1.3. For any permutation $\varphi$ of $\{1, \ldots, n\}$, the orbit $\left(s_{\varphi(1)}, \ldots, s_{\varphi(n)}\right) \cdot B_{n}$ of the element $\left(s_{\varphi(1)}, \ldots, s_{\varphi(n)}\right)$ consists of $(n+1)^{n-1}$ elements.

In Section 2, we prepare some notions which are used later. Section 3 is devoted to the proof of Theorem 3.2 which is a generalization of Theorem 1.3.

Throughout this paper, $n$ is an integer with $n \geq 2$.

2. Some notions. Throughout this section, A is a fixed subset of $\{2, \ldots, n\}$. For integers $i$ and $j$ with $1 \leq i<j \leq n+1$, we define $s_{i j}^{\mathrm{A}} \in B_{n+1}$ by

$$
s_{i j}^{\mathrm{A}}=\left(\prod_{k=i+1}^{j-1}\left(s_{k}\right)^{\epsilon_{k}}\right)^{-1} s_{i} \prod_{k=i+1}^{j-1}\left(s_{k}\right)^{\epsilon_{k}},
$$

where $\epsilon_{k}=1$ if $k \in \mathrm{A}$ and $\epsilon_{k}=-1$ if $k \notin \mathrm{A}$ (see Example 2.1(1)).

We call $s_{i j}^{\mathrm{A}}$ a band generator of $B_{n+1}$ associated with $\mathrm{A}$. Note that a standard generator $s_{i}$ of $B_{n+1}$ is a band generator $s_{i, i+1}^{\mathrm{A}}$.

Let $\Sigma^{\mathrm{A}}$ be the set of band generators $\left\{s_{i j}^{\mathrm{A}} \in B_{n+1} \mid 1 \leq i<j \leq n+1\right\}$ associated with $\mathrm{A}$.

Let $\mathrm{P}_{k}=(k, 0) \in \mathbb{R}^{2}$ for $1 \leq k \leq n+1$. Let $C_{1}$ be the circle in $\mathbb{R}^{2}$ passing through the points $\mathrm{P}_{1}$ and $\mathrm{P}_{n+1}$ with the length of the segment $\overline{\mathrm{P}_{1} \mathrm{P}_{n+1}}$ in diameter. Take the points $\mathrm{Q}_{k} \in C_{1}$ for $1 \leq k \leq n+1$ such that $\mathrm{Q}_{1}=\mathrm{P}_{1}$, $\mathrm{Q}_{n+1}=\mathrm{P}_{n+1}$ and $\mathrm{Q}_{k}=\left(k, y_{k}\right)$ for each $2 \leq k \leq n$, where $y_{k}<0$ if $k \in \mathrm{A}$ and $y_{k}>0$ if $k \notin \mathrm{A}$.

For $1 \leq i<j \leq n+1$, we call the segment $\overline{\mathrm{Q}_{i} \mathrm{Q}_{j}}$ the segment corresponding to $s_{i j}^{\mathrm{A}}$. (See Example 2.1(2).)

REMARK. The reason to call $\overline{\mathrm{Q}_{i} \mathrm{Q}_{j}}$ the segment corresponding to $s_{i j}^{\mathrm{A}}$ is as follows. 
Let $\mathrm{P}_{0}=\mathrm{Q}_{0}=(0,0) \in \mathbb{R}^{2}$ and $\mathrm{P}_{n+2}=\mathrm{Q}_{n+2}=(n+2,0) \in \mathbb{R}^{2}$. Let $C_{2}$ be the circle in $\mathbb{R}^{2}$ passing through the points $\mathrm{P}_{0}$ and $\mathrm{P}_{n+2}$ with the length of the segment $\overline{\mathrm{P}_{0} \mathrm{P}_{n+2}}$ in diameter. Let $D$ be the disk in $\mathbb{R}^{2}$ with $\partial D=C_{2}$. Take an isotopy $\left\{h_{u}\right\}_{u \in[0,1]}$ of $D$ such that for each $u \in[0,1], h_{0}=\mathrm{id}$, $\left.h_{u}\right|_{\partial D_{n+1}}=\mathrm{id}$, and for each $u \in[0,1]$ and each $(x, y) \in \bigcup_{i=0}^{n+1} \overline{\mathrm{Q}_{i} \mathrm{Q}_{i+1}}$, $h_{u}(x, y)=(x,(1-u) y)$.

Then $h_{1}\left(\mathrm{Q}_{i}\right)=\mathrm{P}_{i}$ for any $i$. For $1 \leq i<j \leq n+1$, we define $\alpha_{i j}^{\mathrm{A}}$ to be the $\operatorname{arc} h_{1}\left(\overline{\mathrm{Q}_{i} \mathrm{Q}_{j}}\right)$ in $D$. Note that $\partial \alpha_{i j}^{\mathrm{A}}=\left\{\mathrm{P}_{i}, \mathrm{P}_{j}\right\}, \alpha_{i j}^{\mathrm{A}}$ is above $\mathrm{P}_{k}$ if $k \in \mathrm{A}$ and $\alpha_{i j}^{\mathrm{A}}$ is below $\mathrm{P}_{k}$ if $k \notin \mathrm{A}$ (see Example 2.1(3)).

The braid group $B_{n+1}$ is isomorphic to the mapping class group of $\left(D,\left\{\mathrm{P}_{1}, \ldots, \mathrm{P}_{n+1}\right\}\right)$ relative to the boundary (cf. [2]).

The band generator $s_{i j}^{\mathrm{A}}$ corresponds to the isotopy class of a homeomorphism from $\left(D,\left\{\mathrm{P}_{1}, \ldots, \mathrm{P}_{n+1}\right\}\right)$ to itself which twists a sufficiently small disk neighborhood of the arc $\alpha_{i j}^{\mathrm{A}}$ by a $180^{\circ}$ clockwise rotation using its collar neighborhood.

By the homeomorphism $h_{1}:\left(D,\left\{\mathrm{Q}_{1}, \ldots, \mathrm{Q}_{n+1}\right\}\right) \rightarrow\left(D,\left\{\mathrm{P}_{1}, \ldots, \mathrm{P}_{n+1}\right\}\right)$, we identify the mapping class group of $\left(D,\left\{\mathrm{Q}_{1}, \ldots, \mathrm{Q}_{n+1}\right\}\right)$ and that of $\left(D,\left\{\mathrm{P}_{1}, \ldots, \mathrm{P}_{n+1}\right\}\right)$. Then the band generator $s_{i j}^{\mathrm{A}}$ corresponds to the isotopy class of a homeomorphism from $\left(D,\left\{\mathrm{Q}_{1}, \ldots, \mathrm{Q}_{n+1}\right\}\right)$ to itself which twists a sufficiently small disk neighborhood of the segment $\overline{Q_{i} Q_{j}}$ by a $180^{\circ}$ clockwise rotation. Therefore, we say that the segment $\overline{\mathrm{Q}_{i} \mathrm{Q}_{j}}$ corresponds to the band generator $s_{i j}^{\mathrm{A}} \in \Sigma^{\mathrm{A}}$.

EXAMPle 2.1. Let $n=4$ and $\mathrm{A}=\{2\}$.

(1) The band generator $s_{14}^{\mathrm{A}} \in \Sigma^{\mathrm{A}}$ is $s_{3}\left(s_{2}\right)^{-1} s_{1} s_{2}\left(s_{3}\right)^{-1}$ (see Figure 2.1).

(2) Figure 2.2 shows the segment $\overline{\mathrm{Q}_{1} \mathrm{Q}_{4}}$ corresponding to $s_{14}^{\mathrm{A}} \in \Sigma^{\mathrm{A}}$.

(3) Figure 2.3 shows the $\operatorname{arc} \alpha_{14}^{\mathrm{A}}=h_{1}\left(\overline{\mathrm{Q}_{1} \mathrm{Q}_{4}}\right)$.

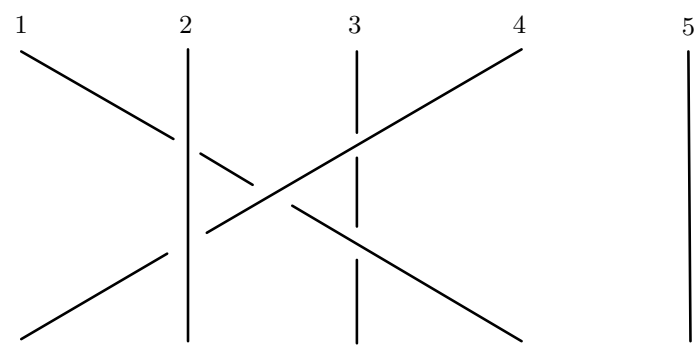

Fig. 2.1

For an element $\left(g_{1}, \ldots, g_{n}\right)$ of the $n$-fold product $\left(\Sigma^{\mathrm{A}}\right)^{n}$ of $\Sigma^{\mathrm{A}}$, we call an $n$-tuple $\left(a_{1}, \ldots, a_{n}\right)$ of the segments $a_{i}$ corresponding to $g_{i}$ the segment system corresponding to $\left(g_{1}, \ldots, g_{n}\right)$. 


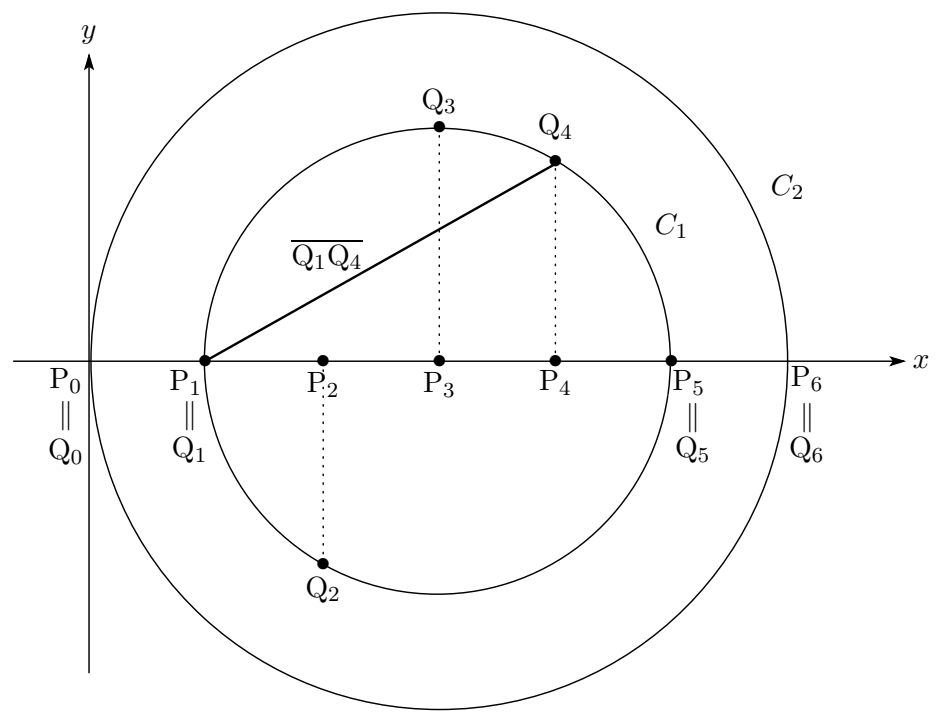

Fig. 2.2. $n=4$ and $\mathrm{A}=\{2\}$

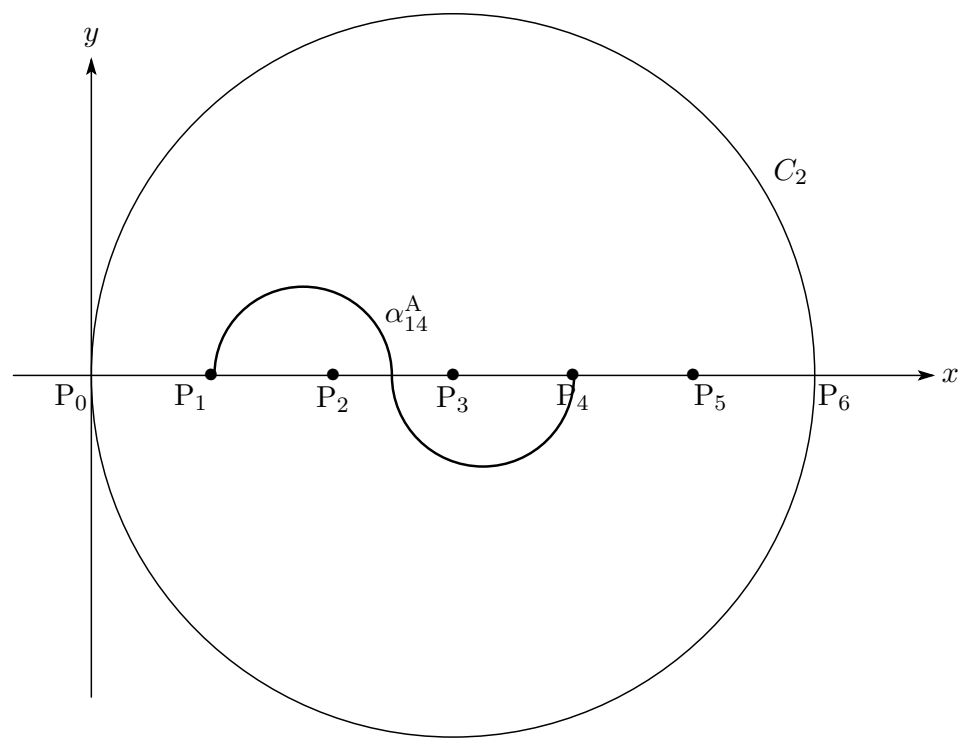

Fig. 2.3

Let $a$ and $a^{\prime}$ be the segments corresponding to elements $g$ and $g^{\prime}$ of $\Sigma^{\mathrm{A}}$. If $\partial a=\left\{\mathrm{Q}_{i}, \mathrm{Q}_{i^{\prime}}\right\}, \partial a^{\prime}=\left\{\mathrm{Q}_{i}, \mathrm{Q}_{i^{\prime \prime}}\right\}$ and $\mathrm{Q}_{i^{\prime}} \neq \mathrm{Q}_{i^{\prime \prime}}$, i.e., $a$ and $a^{\prime}$ share a common end point $\mathrm{Q}_{i}$, then we say that $a$ and $a^{\prime}$ are adjacent (at $\mathrm{Q}_{i}$ ). Moreover, if the end points $\mathrm{Q}_{i^{\prime}}, \mathrm{Q}_{i}$ and $\mathrm{Q}_{i^{\prime \prime}}$ appear on $C_{1}$ counterclockwise in this order, then we say that $a^{\prime}$ is right adjacent to $a$ (at $\mathrm{Q}_{i}$ ), or $a$ is left adjacent to $a^{\prime}$. 
Definition 2.2. An element $\left(g_{1}, \ldots, g_{n}\right)$ of $\left(\Sigma^{\mathrm{A}}\right)^{n}$ is A-good if the segment system $\left(a_{1}, \ldots, a_{n}\right)$ corresponding to $\left(g_{1}, \ldots, g_{n}\right)$ satisfies the following conditions:

(i) If $k \neq l$, then $a_{k}$ and $a_{l}$ are disjoint or adjacent,

(ii) If $k<l$ and $a_{k}$ and $a_{l}$ intersect, then $a_{l}$ is right adjacent to $a^{\prime}$.

(iii) The union $a_{1} \cup \cdots \cup a_{n}$ is a tree as a graph.

ExAmple 2.3. Let $n=4$ and $\mathrm{A}=\{2\}$. Then $\left(s_{23}^{\mathrm{A}}, s_{24}^{\mathrm{A}}, s_{13}^{\mathrm{A}}, s_{25}^{\mathrm{A}}\right)$ is $\mathrm{A}$ good. The segments $a_{1}, \ldots, a_{4}$ corresponding to $s_{23}^{\mathrm{A}}, s_{24}^{\mathrm{A}}, s_{13}^{\mathrm{A}}, s_{25}^{\mathrm{A}}$ are depicted in Figure 2.4.

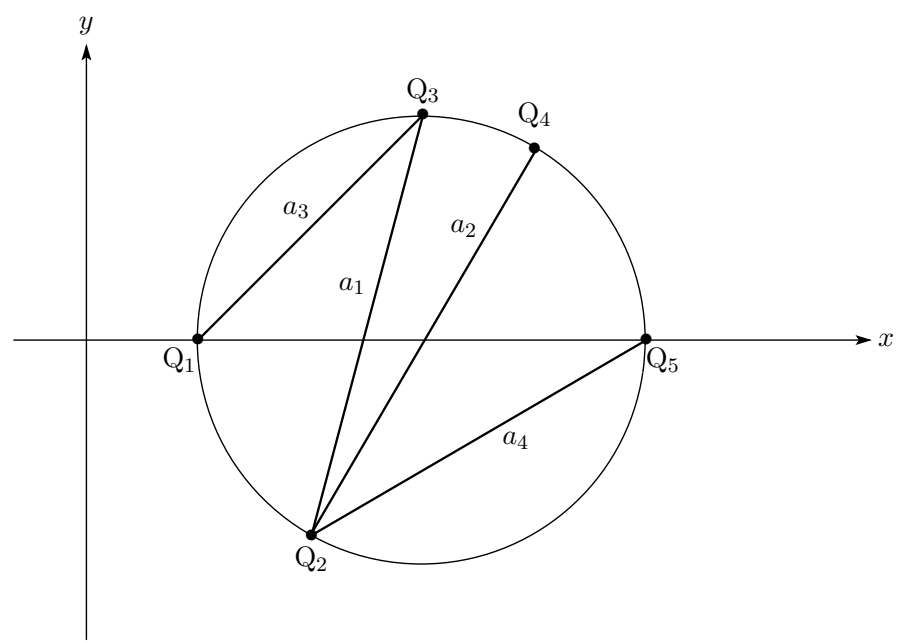

Fig. 2.4

Let $\left(g_{1}, \ldots, g_{n}\right)$ be an element of $\left(\Sigma^{\mathrm{A}}\right)^{n}$ that is A-good and let $\left(a_{1}, \ldots, a_{n}\right)$ be the corresponding segment system.

Suppose that $a_{l}$ is right adjacent to $a_{k}$ at $\mathrm{Q}_{i}$ for some $k, l(k<l)$ and some $i$. Put $a_{k}=\overline{\mathrm{Q}_{i} \mathrm{Q}_{i^{\prime}}}$ and $a_{l}=\overline{\mathrm{Q}_{i} \mathrm{Q}_{i^{\prime \prime}}}$. Then the points $\mathrm{Q}_{i^{\prime}}, \mathrm{Q}_{i}$ and $\mathrm{Q}_{i^{\prime \prime}}$ appear counterclockwise in this order and the following lemma holds:

LEMmA 2.4. If $a_{k} \cap a_{m} \cap a_{l}=\left\{\mathrm{Q}_{i}\right\}$ for $m \in\{1, \ldots, n\}, m \neq k, l$, then $a_{m}$ intersects Int $\overline{\mathrm{Q}_{i^{\prime}} \mathrm{Q}_{i^{\prime \prime}}}$ if and only if $k<m<l$. In particular, if $l=k+1$, then $a_{m}$ and Int $\overline{\mathrm{Q}_{i^{\prime}} \mathrm{Q}_{i^{\prime \prime}}}$ are disjoint.

Proof. Put $a_{m}=\overline{\mathrm{Q}_{i} \mathrm{Q}_{j}}$.

(Case I) Suppose that $m<k<l$. Then $a_{m}$ is left adjacent to $a_{k}$ and $a_{l}$ by condition (ii) of Definition 2.2. Hence, the points $\mathrm{Q}_{j}, \mathrm{Q}_{i}, \mathrm{Q}_{i^{\prime \prime}}$ and $\mathrm{Q}_{i^{\prime}}$ appear counterclockwise in this order. Then $a_{m}$ and Int $\overline{\mathrm{Q}_{i^{\prime}} \mathrm{Q}_{i^{\prime \prime}}}$ are disjoint.

(Case II) Suppose that $k<m<l$. Then $a_{m}$ is right adjacent to $a_{k}$ and left adjacent to $a_{l}$ by condition (ii) of Definition 2.2. Hence, the points $Q_{i^{\prime}}$, 
$\mathrm{Q}_{i}, \mathrm{Q}_{i^{\prime \prime}}$ and $\mathrm{Q}_{j}$ appear counterclockwise in this order. Then $a_{m}$ intersects Int $\overline{\mathrm{Q}_{i^{\prime}} \mathrm{Q}_{i^{\prime \prime}}}$.

(Case III) Suppose that $k<l<m$. Then $a_{m}$ is right adjacent to $a_{k}$ and $a_{l}$ by condition (ii) of Definition 2.2. Hence, the points $\mathrm{Q}_{i^{\prime}}, \mathrm{Q}_{i}, \mathrm{Q}_{j}$ and $\mathrm{Q}_{i^{\prime \prime}}$ appear counterclockwise in this order. Then $a_{m}$ and Int $\overline{\mathrm{Q}_{i^{\prime}} \mathrm{Q}_{i^{\prime \prime}}}$ are disjoint.

Thus, $a_{m}$ intersects Int $\overline{\mathrm{Q}_{i^{\prime}} \mathrm{Q}_{i^{\prime \prime}}}$ if and only if $k<m<l$.

3. Proof of Theorem 1.3. The following lemma is the first step towards the proof of Theorem 1.3.

Lemma 3.1. For an element $\varphi \in \operatorname{Sym}\{1, \ldots, n\}$, let $\mathrm{A}=\{i \in \mathbb{N}$ $\left.\varphi^{-1}(i-1)<\varphi^{-1}(i), 2 \leq i \leq n\right\}$. Then $\left(s_{\varphi(1)}, \ldots, s_{\varphi(n)}\right)$ is an element of $\left(\Sigma^{\mathrm{A}}\right)^{n}$ and it is A-good.

Proof. Since the standard generators of $B_{n+1}$ belong to $\Sigma^{\mathrm{A}}$, it follows that $\left(s_{\varphi(1)}, \ldots, s_{\varphi(n)}\right) \in\left(\Sigma^{\mathrm{A}}\right)^{n}$. The arc $a_{m}$ corresponding to $s_{\varphi(m)}$ is $\overline{\mathrm{Q}_{\varphi(m)} \mathrm{Q}_{\varphi(m)+1}}$. Suppose that $a_{k} \cap a_{l} \neq \emptyset$ for $k<l$. Then $a_{k} \cap a_{l}=\left\{\mathrm{Q}_{\varphi(k)}\right\}$ or $\left\{\mathrm{Q}_{\varphi(l)}\right\}$, and $|\varphi(k)-\varphi(l)|=1$. Assume $\varphi(l)-\varphi(k)=1$, so that $a_{k} \cap a_{l}=$ $\left\{\mathrm{Q}_{\varphi(l)}\right\}$ and $\varphi(l) \in \mathrm{A}$. Then the points $\mathrm{Q}_{\varphi(k)}, \mathrm{Q}_{\varphi(l)}$ and $\mathrm{Q}_{\varphi(l)+1}$ appear counterclockwise in this order since the $y$-coordinate of $\mathrm{Q}_{\varphi(l)}$ is negative. Thus, $a_{l}$ is right adjacent to $a_{k}$. Assume $\varphi(k)-\varphi(l)=1$, so that $a_{k} \cap a_{l}=\left\{\mathrm{Q}_{\varphi(k)}\right\}$ and $\varphi(k) \notin \mathrm{A}$. Then the points $\mathrm{Q}_{\varphi(k+1)}, \mathrm{Q}_{\varphi(k)}$ and $\mathrm{Q}_{\varphi(l)}$ appear counterclockwise in this order since the $y$-coordinate of $\mathrm{Q}_{\varphi(l)}$ is positive. Thus, $a_{l}$ is right adjacent to $a_{k}$. We easily see that the graph $a_{1} \cup \cdots \cup a_{n}$ is a tree.

Theorem 1.3 is obtained from the following theorem by Lemma 3.1.

Theorem 3.2. Let A be a subset of $\{2, \ldots, n\}$. For any element $\left(g_{1}, \ldots, g_{n}\right) \in\left(\Sigma^{\mathrm{A}}\right)^{n}$ that is A-good, the orbit $\left(g_{1}, \ldots, g_{n}\right) \cdot B_{n}$ consists of $(n+1)^{n-1}$ elements.

The rest of this paper is devoted to proving Theorem 3.2.

For $\left(g_{1}, \ldots, g_{n}\right) \in\left(\Sigma^{\mathrm{A}}\right)^{n}$, it is not always the case that $\left(g_{1}, \ldots, g_{n}\right) \cdot B_{n}$ $\subset\left(\Sigma^{\mathrm{A}}\right)^{n}$. However, we have

Lemma 3.3. Let $\mathrm{A}$ be a subset of $\{2, \ldots, n\}$. If $\left(g_{1}, \ldots, g_{n}\right) \in\left(\Sigma^{\mathrm{A}}\right)^{n}$ is A-good, then, for any $k \in\{1, \ldots, n-1\}$ and any $\epsilon \in\{1,-1\}$, we have:

(1) $\left(g_{1}, \ldots, g_{n}\right) \cdot\left(\sigma_{k}\right)^{\epsilon} \in\left(\Sigma^{\mathrm{A}}\right)^{n}$,

(2) $\left(g_{1}, \ldots, g_{n}\right) \cdot\left(\sigma_{k}\right)^{\epsilon}$ is A-good.

Proof. Let $\left(a_{1}, \ldots, a_{n}\right)$ be the segment system corresponding to $\left(g_{1}, \ldots, g_{n}\right)$, and let $\left(b_{1}, \ldots, b_{n}\right)$ be that corresponding to $\left(g_{1}, \ldots, g_{n}\right) \cdot \sigma_{k}$.

First we consider the case where $a_{k}$ and $a_{k+1}$ are disjoint. Then $g_{k}$ and $g_{k+1}$ are commutative, and 


$$
\begin{aligned}
\left(g_{1}, \ldots, g_{k-1}, g_{k}, g_{k+1},\right. & \left.g_{k+2}, \ldots, g_{n}\right) \cdot \sigma_{k} \\
& =\left(g_{1}, \ldots, g_{k-1}, g_{k}, g_{k+1}, g_{k+2}, \ldots, g_{n}\right) \cdot\left(\sigma_{k}\right)^{-1} \\
& =\left(g_{1}, \ldots, g_{k-1}, g_{k+1}, g_{k}, g_{k+2}, \ldots, g_{n}\right) .
\end{aligned}
$$

Thus, we obtain (1).

For the proof of (2), it is enough to prove $\left(g_{1}, \ldots, g_{n}\right) \cdot \sigma_{k}$ is A-good. Since $b_{k}=a_{k+1}, b_{k+1}=a_{k}$ and $b_{p}=a_{p}$ for $p \neq k, k+1$, we see that $\left(g_{1}, \ldots, g_{n}\right) \cdot \sigma_{k}$ satisfies conditions (i) and (iii) of Definition 2.2. Suppose that $b_{p}$ and $b_{q}$ intersect for some $p$ and $q(p<q)$. Let $b_{p}=a_{p^{\prime}}, b_{q}=a_{q^{\prime}}$. Note that $(p, q) \neq(k, k+1)$ and $\left(p^{\prime}, q^{\prime}\right) \neq(k+1, k)$ because $b_{k} \cap b_{k+1}=a_{k+1} \cap a_{k}=\emptyset$. Thus, we have $p^{\prime}<q^{\prime}$ because $p<q$. Since $a_{p^{\prime}}$ and $a_{q^{\prime}}$ satisfy condition (ii) of Definition 2.2, so do $b_{p}$ and $b_{q}$. We have (2).

Now consider the case where $a_{k}$ and $a_{k+1}$ intersect. Let $\mathrm{Q}_{x}, \mathrm{Q}_{y}, \mathrm{Q}_{z}$ $(x<y<z)$ be the points such that $\left\{\mathrm{Q}_{x}, \mathrm{Q}_{y}, \mathrm{Q}_{z}\right\}=\partial a_{k} \cup \partial a_{k+1}$.

By condition (ii) of Definition 2.2, $a_{k}$ and $a_{k+1}$ satisfy one of the following conditions:

(A1) $y \in \mathrm{A}$ and $a_{k}=\overline{\mathrm{Q}_{x} \mathrm{Q}_{y}}, a_{k+1}=\overline{\mathrm{Q}_{y} \mathrm{Q}_{z}}$,

(A2) $y \in \mathrm{A}$ and $a_{k}=\overline{\mathrm{Q}_{y} \mathrm{Q}_{z}}, a_{k+1}=\overline{\mathrm{Q}_{x} \mathrm{Q}_{z}}$,

(A3) $y \in \mathrm{A}$ and $a_{k}=\overline{\mathrm{Q}_{x} \mathrm{Q}_{z}}, a_{k+1}=\overline{\mathrm{Q}_{x} \mathrm{Q}_{y}}$,

(A4) $y \notin \mathrm{A}$ and $a_{k}=\overline{\mathrm{Q}_{y} \mathrm{Q}_{z}}, a_{k+1}=\overline{\mathrm{Q}_{x} \mathrm{Q}_{y}}$,

(A5) $y \notin \mathrm{A}$ and $a_{k}=\overline{\mathrm{Q}_{x} \mathrm{Q}_{y}}, a_{k+1}=\overline{\mathrm{Q}_{x} \mathrm{Q}_{z}}$,

(A6) $y \notin \mathrm{A}$ and $a_{k}=\overline{\mathrm{Q}_{x} \mathrm{Q}_{z}}, a_{k+1}=\overline{\mathrm{Q}_{y} \mathrm{Q}_{z}}$.

Then $\left(g_{k}, g_{k+1}\right)=\left(s_{x y}^{\mathrm{A}}, s_{y z}^{\mathrm{A}}\right),\left(s_{y z}^{\mathrm{A}}, s_{x z}^{\mathrm{A}}\right),\left(s_{x z}^{\mathrm{A}}, s_{x y}^{\mathrm{A}}\right),\left(s_{y z}^{\mathrm{A}}, s_{x y}^{\mathrm{A}}\right),\left(s_{x y}^{\mathrm{A}}, s_{x z}^{\mathrm{A}}\right)$ or $\left(s_{x z}^{\mathrm{A}}, s_{y z}^{\mathrm{A}}\right)$. By direct calculations $\left(g_{k+1},\left(g_{k+1}\right)^{-1} g_{k} g_{k+1}\right)=\left(s_{y z}^{\mathrm{A}}, s_{x z}^{\mathrm{A}}\right)$, $\left(s_{x z}^{\mathrm{A}}, s_{x y}^{\mathrm{A}}\right),\left(s_{x y}^{\mathrm{A}}, s_{y z}^{\mathrm{A}}\right),\left(s_{x y}^{\mathrm{A}}, s_{x z}^{\mathrm{A}}\right),\left(s_{x z}^{\mathrm{A}}, s_{y z}^{\mathrm{A}}\right)$ or $\left(s_{y z}^{\mathrm{A}}, s_{x y}^{\mathrm{A}}\right)$, respectively. This implies that $\left(g_{1}, \ldots, g_{n}\right) \cdot \sigma_{k}$ and $\left(g_{1}, \ldots, g_{n}\right) \cdot\left(\sigma_{k}\right)^{2}$ are elements of $\left(\Sigma^{\mathrm{A}}\right)^{n}$ and $\left(g_{1}, \ldots, g_{n}\right) \cdot\left(\sigma_{k}\right)^{3}=\left(g_{1}, \ldots, g_{n}\right)$. Note that $\left(g_{1}, \ldots, g_{n}\right) \cdot\left(\sigma_{k}\right)^{-1} \in\left(\Sigma_{\mathrm{A}}\right)^{n}$ since $\left(g_{1}, \ldots, g_{n}\right) \cdot\left(\sigma_{k}\right)^{-1}=\left(g_{1}, \ldots, g_{n}\right) \cdot\left(\sigma_{k}\right)^{2}$. Thus, we obtain (1).

For (2), it is sufficient to prove $\left(g_{1}, \ldots, g_{n}\right) \cdot \sigma_{k}$ is A-good. Note that $b_{k}=a_{k+1}, b_{k+1}$ is the edge of the boundary of $\left|\mathrm{Q}_{x} \mathrm{Q}_{y} \mathrm{Q}_{z}\right|$ that is neither $a_{k}$ nor $a_{k+1}$, and $b_{p}=a_{p}$ for $p \neq k, k+1$. Thus, we see that $b_{p}$ and $b_{k}$ are disjoint or $b_{p} \cap b_{k}=\left\{Q_{i}\right\}$ for $p \neq k, k+1$ and some $i$, and $b_{p}$ and $b_{q}$ are disjoint or $b_{p} \cap b_{q}=\left\{\mathrm{Q}_{i}\right\}$ for $p \neq q \in\{1, \ldots, n\} \backslash\{k, k+1\}$ and some $i$. By Lemma 2.4, for $p \neq k, k+1, a_{p}$ and Int $b_{k+1}$ are disjoint. Thus, $b_{p}$ and $b_{k+1}$ are disjoint or $b_{p} \cap b_{k+1}=\left\{\mathrm{Q}_{i}\right\}$ for $p \neq k, k+1$ and some $i$, and $\left(g_{1}, \ldots, g_{n}\right) \cdot \sigma_{k}$ satisfies condition (i) of Definition 2.2.

Let $X$ be the space defined by

$$
\begin{aligned}
X & =a_{1} \cup \cdots \cup a_{k-1} \cup\left|\mathrm{Q}_{x} \mathrm{Q}_{y} \mathrm{Q}_{z}\right| \cup a_{k+2} \cup \cdots \cup a_{n} \\
& =b_{1} \cup \cdots \cup b_{k-1} \cup\left|\mathrm{Q}_{x} \mathrm{Q}_{y} \mathrm{Q}_{z}\right| \cup b_{k+2} \cup \cdots \cup b_{n} .
\end{aligned}
$$


Note that $X$ is homotopy equivalent to $a_{1} \cup \cdots \cup a_{n}$ and $b_{1} \cup \cdots \cup b_{n}$. Since $a_{1} \cup \cdots \cup a_{n}$ is a tree, we see that $b_{1} \cup \cdots \cup b_{n}$ is a tree. Thus, $\left(g_{1}, \ldots, g_{n}\right) \cdot \sigma_{k}$ satisfies condition (iii) of Definition 2.2.

We have already seen that $b_{k}$ and $b_{k+1}$ satisfy condition (A2), (A3), (A1), (A5), (A6) or (A4) if $a_{k}$ and $a_{k+1}$ satisfy (A1), (A2), (A3), (A4), (A5) or (A6), respectively. Let $p \neq q \in\{1, \ldots, n\} \backslash\{k, k+1\}$. If $b_{p}\left(=a_{p}\right)$ and $b_{q}$ $\left(=a_{q}\right)$ intersect, then they satisfy condition (ii) of Definition 2.2. If $b_{k}$ and $b_{p}$ intersect, then $b_{k}=a_{k+1}$ and $b_{p}=a_{p}$ satisfy condition (ii) of Definition 2.2 since $p<k$ iff $p<k+1$.

The remainder of the proof of (2) is to check that $b_{k+1}$ and $b_{p}$ satisfy condition (ii) of Definition 2.2 if $b_{k+1}$ and $b_{p}$ intersect for $p \in\{1, \ldots, n\} \backslash$ $\{k, k+1\}$.

Let $b_{k} \cap b_{k+1}=\left\{\mathrm{Q}_{i}\right\}, b_{k}=\overline{\mathrm{Q}_{i} \mathrm{Q}_{i^{\prime}}}$ and $b_{k+1}=\overline{\mathrm{Q}_{i} \mathrm{Q}_{i^{\prime \prime}}}$. Then we have already seen that $a_{k}=\overline{\mathrm{Q}_{i^{\prime}} \mathrm{Q}_{i^{\prime \prime}}}, a_{k+1}=b_{k}=\overline{\mathrm{Q}_{i} \mathrm{Q}_{i^{\prime}}}$ and the points $\mathrm{Q}_{i^{\prime \prime}}, \mathrm{Q}_{i^{\prime}}$ and $\mathrm{Q}_{i}$ appear counterclockwise in this order.

(Case 1) Suppose that $b_{p}$ is adjacent to $b_{k+1}$ at $\mathrm{Q}_{i}$ and let $b_{p}=\overline{\mathrm{Q}_{i} \mathrm{Q}_{j}}$.

(Case 1-1) Suppose that $p<k$. Then we have seen that $b_{p}$ is left adjacent to $b_{k}$. Since $b_{k}$ is left adjacent to $b_{k+1}$, we see that $b_{p}$ is left adjacent to $b_{k+1}$.

(Case 1-2) Suppose that $p>k$. Then we have seen that $b_{p}$ is right adjacent to $b_{k}$ and the points $\mathrm{Q}_{i^{\prime}}, \mathrm{Q}_{i}$ and $\mathrm{Q}_{j}$ appear counterclockwise in this order. By Lemma 2.4, the points $\mathrm{Q}_{i^{\prime \prime}}, \mathrm{Q}_{i}$ and $\mathrm{Q}_{j}$ appear counterclockwise in this order. Thus, $b_{p}$ is right adjacent to $b_{k+1}$.

(Case 2) Suppose that $b_{p}$ is adjacent to $b_{k+1}$ at $\mathrm{Q}_{i^{\prime \prime}}$ and let $b_{p}=\overline{\mathrm{Q}_{i^{\prime \prime}} \mathrm{Q}_{j}}$.

(Case 2-1) Suppose that $p<k$. Then we have seen that $b_{p}\left(=a_{p}\right)$ is right adjacent to $\overline{\mathrm{Q}_{i^{\prime}} \mathrm{Q}_{i^{\prime \prime}}}=a_{k}$ at $\mathrm{Q}_{i^{\prime \prime}}$ by condition (ii) of Definition 2.2. Thus, $\mathrm{Q}_{j}, \mathrm{Q}_{i^{\prime \prime}}$ and $\mathrm{Q}_{i^{\prime}}$ appear counterclockwise in this order. By Lemma 2.4, $\mathrm{Q}_{j}$, $\mathrm{Q}_{i^{\prime \prime}}$ and $\mathrm{Q}_{i}$ appear counterclockwise in this order. Thus, $b_{p}$ is left adjacent to $b_{k+1}$.

(Case 2-2) Suppose that $p>k$. Then we have seen that $b_{p}=a_{p}$ is right adjacent to $\overline{\mathrm{Q}_{i^{\prime}} \mathrm{Q}_{i^{\prime \prime}}}=a_{k}$ at $\mathrm{Q}_{i^{\prime \prime}}$ by condition (ii) of Definition 2.2. Note that $a_{k}$ is right adjacent to $b_{k+1}$. Thus, $b_{p}$ is right adjacent to $b_{k+1}$.

Consequently, $b_{k+1}$ and $b_{p}$ satisfy condition (ii) of Definition 2.2 in the case where $b_{k+1}=\overline{\mathrm{Q}_{x} \mathrm{Q}_{z}}$, and this completes the proof of Lemma 3.3.

Let $S_{n+1}$ be the symmetric group of degree $n+1$.

LEMma 3.4 ([6]). Let $\tau_{1}, \ldots, \tau_{n}$ be the transpositions in $S_{n+1}$ satisfying $\tau_{i} \neq \tau_{j}(i \neq j)$. Then the orbit of $\left(\tau_{1}, \ldots, \tau_{n}\right)$ under the Hurwitz action of $B_{n}$ on $\left(S_{n+1}\right)^{n}$ consists of $(n+1)^{n-1}$ elements.

For groups $G, H$ and a homomorphism $f: G \rightarrow H$, let $f^{n}: G^{n} \rightarrow H^{n}$ be the map defined by $\left(g_{1}, \ldots, g_{n}\right) \mapsto\left(f\left(g_{1}\right), \ldots, f\left(g_{n}\right)\right)$. The following lemma is easily seen. 
Lemma 3.5. For any $\beta \in B_{n}$,

$$
f^{n}\left(\left(g_{1}, \ldots, g_{n}\right) \cdot \beta\right)=\left(f^{n}\left(g_{1}, \ldots, g_{n}\right)\right) \cdot \beta \text {. }
$$

Proof of Theorem 3.2. Note that the restriction $\left.p\right|_{\Sigma^{\mathrm{A}}}$ of the canonical projection $p: B_{n+1} \rightarrow S_{n+1}$ to $\Sigma^{\mathrm{A}}$ is injective and the image $p\left(\Sigma^{\mathrm{A}}\right)$ is the set of all transpositions of $S_{n+1}$. By Lemma 3.3 , we see $\left(g_{1}, \ldots, g_{n}\right) \cdot B_{n} \subset$ $\left(\Sigma^{\mathrm{A}}\right)^{n}$. Hence, $\#\left(\left(g_{1}, \ldots, g_{n}\right) \cdot B_{n}\right)=\#\left(p^{n}\left(\left(g_{1}, \ldots, g_{n}\right) \cdot B_{n}\right)\right)$. By Lemma $3.5, \#\left(p^{n}\left(\left(g_{1}, \ldots, g_{n}\right)\right) \cdot B_{n}\right)=\#\left(\left(p^{n}\left(g_{1}, \ldots, g_{n}\right)\right) \cdot B_{n}\right)$. By the definition of A-good, $g_{k} \neq g_{l}$ for $k \neq l$ (since the arcs $a_{k}$ and $a_{l}$ corresponding to $g_{k}$ and $g_{l}$ are disjoint or they meet only in their end point). Hence, $p^{n}\left(g_{1}, \ldots, g_{n}\right)$ is an element whose components are mutually distinct transpositions of $S_{n+1}$. By Lemma 3.4, we obtain the result.

Acknowledgments. The author would like to thank Professors Takao Matumoto, Seiichi Kamada and Makoto Sakuma for their help. He is partly supported by JSPS Research Fellowships for Young Scientists.

\section{References}

[1] E. Artin, Theorie der Zöpfe, Abh. Math. Sem. Univ. Hamburg 4 (1925), 47-72.

[2] J. Birman, Braids, Links, and Mapping Class Groups, Ann. of Math. Stud. 82, Princeton Univ. Press, 1974.

[3] F. Bohnenblust, On algebraic braid groups, Ann. of Math. 48 (1947), 127-136.

[4] S. P. Humphries, Finite Hurwitz braid group actions for Artin groups, Israel J. Math. 143 (2004), 189-222.

[5] S. Kamada, Braid and Knot Theory in Dimension Four, Math. Surveys Monogr. 95, Amer. Math. Soc., 2002.

[6] P. Kluitmann, Hurwitz action and finite quotients of braid groups, in: Contemp. Math. 78, Amer. Math. Soc., 1988, 299-325.

[7] B. G. Moishezon, Stable branch curves and braid monodromies, in: Algebraic Geometry, Lecture Notes in Math. 862, Springer, 1981, 107-192.

[8] B. Moishezon and M. Teicher, Braid group technique in complex geometry I. Line arrangements in $\mathbb{C P}^{2}$, in: Contemp. Math. 78, Amer. Math. Soc., 1988, 425-555.

[9] L. Rudolph, Braided surfaces and Seifert ribbons for closed braids, Comment. Math. Helv. 58 (1983), 1-37.

[10] Y. Yaguchi, Isotropy subgroup of Hurwitz action of the 3-braid group on the braid systems, J. Knot Theory Ramif. 18 (2009), 1021-1030.

Yoshiro Yaguchi

Department of Mathematics

Hiroshima University

Higashi-Hiroshima, 739-8526 Japan

E-mail: d083645@hiroshima-u.ac.jp 